Koeijers, J.J., Verbon, A., Kessels, A.G.H., Bartelds, A., Donker, G., Nys, S., Stobberingh, E.E. Urinary tract infection in male general practice patients: uropathogens and antibiotic susceptibility. Urology: 2010, 76(2), 336-340

\begin{tabular}{|c|c|}
\hline $\begin{array}{l}\text { Postprint } \\
\text { Version }\end{array}$ & 1.0 \\
\hline Journal website & http://dx.doi.org/10.1016/j.urology.2010.02.052 \\
\hline Pubmed link & http://www.ncbi.nlm.nih.gov/pubmed/20494416 \\
\hline DOI & 10.1016/j.urology.2010.02.052 \\
\hline
\end{tabular}

This is a NIVEL certified Post Print, more info at http://www.nivel.eu

\title{
Urinary Tract Infection in Male General Practice Patients: Uropathogens and Antibiotic Susceptibility
}

\author{
J.J. KoEIJERS ${ }^{\text {A, }}$ A. VERBON ${ }^{\mathrm{A}}$, A.G.H. KeSSELS ${ }^{\mathrm{A}}$, A. BARTELDS ${ }^{\mathrm{A}}$, G. DONKERS ${ }^{\mathrm{A}}$, S. NYS ${ }^{\mathrm{A}}$ AND E.E. \\ STOBBERINGH $^{\mathrm{A}}$ \\ ${ }^{a}$ Departments of Internal Medicine/Department of Infectious Disease, Clinical Epidemiology and MTA, and \\ Medical Microbiology, University Hospital Maastricht, Maastricht, The Netherlands; NIVEL, Netherlands \\ Institute for Health Services Research, Utrecht, The Netherlands; and Department of Internal Medicine, \\ Saint Elisabeth Hospital, Curaçao, Netherlands Antilles
}

OBJECTIVES: To evaluate uropathogens and their antibiotic susceptibility in male general practitioner (GP) patients presenting with an uncomplicated urinary tract infection (UTI).

MATERIAL AND METHODS: A population-based study was conducted among males, 18 years and older, general practice patients, who had symptoms indicative of an uncomplicated UTI. A UTI was defined as $\geq 10^{3}$ colony-forming units $/ \mathrm{mL}(\mathrm{CFU} / \mathrm{mL})$. The etiology of the infection, antimicrobial susceptibility, and treatment strategies used by the GP were determined.

RESULTS: Escherichia coli was most frequently isolated (48\%), followed by other enterobacteriaceae (24\%) and enterococci (9\%). The etiology of infection was age-dependent; E. coli was more frequently isolated in younger patients and Pseudomonas aeruginosa in the elderly. The overall susceptibility rates were low for amoxicillin (63\%) and trimethoprim (70\%), and high for fluoroquinolones (91\%) and amoxicillin-clavulanic acid (90\%), which is similar to susceptibility rates in females with UTIs from the same population. Antibiotics were prescribed to $59 \%$ of the males with symptoms of UTI. Fluoroquinolones were given to $33 \%$ of the patients and trimethoprim-sulfamethoxazole to $24 \%$. No difference in antibiotic prescription, nor in duration of therapy, was found between the different age groups.

CONCLUSIONS: In the male presenting with complaints of an acute uncomplicated UTI at the GP, E. coli, followed by other Gram-negative bacteria were the most frequently isolated uropathogens. Susceptibility rates in uncomplicated male and female UTIs were similar, indicating that data from UTI susceptibility studies in females from the same geographic region can be useful in the choice of empirical therapy in males.

Knowledge of male urinary tract infection (UTI) is less than that of female UTI, despite the fact that onethird of all 80 -year-old men will have had an episode of bacteriuria. ${ }^{[1]}$ and ${ }^{[2]}$ The pathogenesis of uncomplicated UTI in men remains unclear, and it has been hypothesized that all UTI in men must be considered complicated because they result from an anatomic or functional anomaly. There is, however, little evidence to support this hypothesis. ${ }^{[1]}$ and ${ }^{[2]}$ Twenty percent of diagnosed uncomplicated UTI occur in men, with the prevalence escalating with increasing age. ${ }^{3}$ Most male UTIs are seen and treated by the general practitioner (GP). ${ }^{[4]}$ and [5]

Females with uncomplicated UTIs are managed effectively and safely by empirical antibiotic therapy without the need for a urine culture, but the diagnosis of uncomplicated UTI in men is still difficult. Therefore, in male patients, culture and targeted antibiotic therapy has been advocated before a treatment 
Koeijers, J.J., Verbon, A., Kessels, A.G.H., Bartelds, A., Donker, G., Nys, S., Stobberingh, E.E. Urinary tract infection in male general practice patients: uropathogens and antibiotic susceptibility. Urology: 2010

decision is made, ${ }^{5}$ although use of the nitrite dipstick method has been advocated and may result in earlier start of therapy. ${ }^{6}$ Empirical antibiotic subscription by GPs should be based on the availability of actual antibiotic resistance data of the bacterial population to be treated. Unfortunately, most of the antibiotic resistance patterns are derived from uropathogens isolated from female patients. ${ }^{[7],[8],[9] \text { and }[10]}$ Uropathogens causing UTIs in different age groups of male patients and the antibiotic susceptibility of the microorganisms involved are hardly available. ${ }^{[5],[11] \text { and }[12]}$ Here, we evaluated uropathogens and their antibiotic susceptibility in male GP patients presenting with an uncomplicated UTI.

\section{MATERIALS AND METHODS}

\section{Population}

From January 2003 to December 2004, 21 general practices participating in the NIVEL (Netherlands Institute for Health Services Research) sentinel project, monitored personal, diagnostic, and therapeutic information of consecutive male patients 18 years and older, with symptoms indicative of a UTI (ie, acute signs of dysuria, urinary frequency, and/or urgency without the presence of temperature $>38^{\circ} \mathrm{C}$ ), without systemic complaints, foreign body, history of urological complaints, urine catheter, or suspicion of sexually transmitted disease. The design of the study did not differentiate between an uncomplicated UTI or the first UTI developing to a complicated or recurrent course. The patient population of these practices accounts for approximately $1 \%$ of the Dutch population and was representative for age, gender, regional distribution, and degree of urbanization. All patients received care as usual, ie, diagnostic tests and empirical therapy according to the daily practice of the GP.

\section{Urine Sampling and Processing}

Male patients suspected of having a UTI provided a fresh voided (midstream) urine sample as part of usual care. Subsequently, a dipslide (Uriline, 56 508, bioMérieux, Plainview, NY) was performed according to the manufacturer's instructions and sent by mail to the laboratory of Medical Microbiology of the University Hospital Maastricht, The Netherlands, for identification and antibiotic susceptibility testing of the uropathogens. On the day of arrival, the dipslide was incubated at $37^{\circ} \mathrm{C}$ overnight. The growth of bacteria was recorded according to the manufacturer's instructions. Growth of $\geq 10^{3}$ colony-forming units(CFU)/mL was considered a positive urine culture ${ }^{11}$ and indicative of a UTI in male. The isolated uropathogens were stored at $-70^{\circ} \mathrm{C}$ for further analysis. In case of more than one cultured bacteria, only the predominant microorganism was counted.

\section{Antimicrobial Susceptibility}

Minimum inhibitory concentrations (MICs) were determined according to the NCCLS (CLSI) criteria ${ }^{13}$ using the microbroth dilution method with Mueller Hinton II broth cation adjusted (Becton Dickinson \& Company, Sparks, MD), an inoculum of $5 \times 10^{5} \mathrm{CFU} / \mathrm{mL}$, and overnight incubation at $37^{\circ} \mathrm{C}$. MCS Diagnostics provided the MIC plates with freeze-dried antibiotics (NLDMCS1, MCS Diagnostics BV, Swalmen, The Netherlands). The following antimicrobial agents (range in $\mathrm{mg} / \mathrm{L}$ ) were tested: amoxicillin (0.06-128), amoxicillin-clavulanic acid (0.06-128), trimethoprim (0.03-64), trimethoprim-sulfamethoxazole (0.03-64), norfloxacin (0.03-64), ciprofloxacin (0.008-16), and nitrofurantoin (0.5-512). Breakpoints for susceptibility were according to the NCCLS (CLSI) guidelines. ${ }^{13}$ Norfloxacin, ofloxacin, levofloxacin, and ciprofloxacin were grouped in the fluoroquinolone group.

\section{Statistical Analysis}

For the analysis of the culture results and the susceptibility percentages, the patients were divided in the age groups 18-50, 51-70 and >70 years. For statistical analysis, the program SPSS 15.0 for Windows was used (SPSS, Inc., Chicago, IL). The susceptibility was coded as present or absent and analyzed per uropathogen with the chi-square test.

An outcome was significant if the $P$ value was $>.05$.

\section{RESULTS}

\section{Population and Culture Results}

In total, 422 male patients were included in the study. The age of the patients ranged from 18-104 years, with mean and median ages of 58 years. A positive urine culture was found in $236(56 \%)$ men (mean age 60 years, median age 62 years). Positive urine cultures increased with age; 68 of 150 patients in the age 
Koeijers, J.J., Verbon, A., Kessels, A.G.H., Bartelds, A., Donker, G., Nys, S., Stobberingh, E.E. Urinary tract infection in male general practice patients: uropathogens and antibiotic susceptibility. Urology: 2010

category of $18-50$ years $(45 \%)$ had a positive urine culture. In the age category of 51-70 years, 90 of 163 patients $(55 \%)$ had a positive urine culture, and in the age category $>70$ years, 78 of 109 men $(72 \%)$ had a positive urine culture $(P=.03)$. In most of the samples $(91 \%)$, one species was cultured. None of the samples were contaminated, ie, contained more than 2 microbial species. Overall, Escherichia coli was the most frequently isolated uropathogen $(48 \%)\left({ }^{\text {Table }}{ }^{1}\right)$.

\section{[TABLE 1.]}

In the different age categories, different predominant uropathogens were cultured $(P=.025)\left({ }^{\text {Table }}{ }^{1}\right) . E$. coli was significantly more isolated in younger patients compared with other Gram-negative bacteria $(P=$ .04 ), and Pseudomonas aeruginosa was more frequently isolated in the elderly compared with other Gramnegative bacteria $(P=.005)$. The mean age of patients with a positive urine culture with $P$. aeruginosa was 73 year, Klebsiella pneumoniae 74 years, Enterococcus faecalis 54 years, and E. coli 59 years.

\section{Antibiotic Susceptibility}

The antibiotic susceptibility of $E$. coli ranged from $75 \%$ for amoxicillin to $100 \%$ for amoxicillinclavulanic acid $\left({ }^{\text {Table } 2}\right.$ ). For both nitrofurantoin and the fluoroquinolones, the susceptibility of $E$. coli was $97 \%$. However, susceptibility for trimethoprim-sulfamethoxazole was only $81 \%$, whereas this drug was prescribed to $24 \%$ of the men ([Table 2] ${ }^{[n d}{ }^{[\text {Table } 3]}$ ).

\section{[TABLE 2. AND TABLE 3.]}

\section{Antibiotic Therapy}

Of all 422 patients, antibiotics were prescribed in $60 \%$ (253 patients). The most frequently prescribed antimicrobial agent in patients of all age groups were fluoroquinolones 33\% (83/253). Its prescription ranged from $35 \%$ in the oldest to $31 \%$ in the younger patients (NS). One-fourth of the patients received trimethoprim-sulfamethoxazole (24\%). Nitrofurantoin and amoxicillin-clavulanic acid were prescribed for $16 \%$ and $11 \%$, respectively $\left({ }^{\text {Table }} 3\right.$ ). Of the patients who received antibiotics, $71 \%$ had a positive urine culture. One-hundred two patients with a positive nitrite test received antibiotics, and 113 patients with a negative nitrite test (NS). The treatment duration was 6-7 days in $44 \%$ but ranged from 3 to $>21$ days $\left({ }^{\text {Fig. }}{ }^{1}\right.$ ). No correlation between duration of antibiotic use and age was found $(P=.64)\left({ }^{\text {Fig. } 1}\right)$.

\section{[Figure 1. ]}

\section{COMMENT}

This study reports on the uropathogens in male patients with symptoms indicative of UTI in a GP population. ${ }^{5}$ The uropathogens that were isolated were mainly Gram-negative, with E. coli as the predominant pathogen. The uropathogens were highly susceptible for amoxicillin-clavulanic acid and for fluoroquinolones.

The frequency of male UTI increases with age, probably secondary to voiding problems. ${ }^{[2],{ }^{[14]} \text { and [15] }}$ Remarkably, symptoms of UTI were more predictive for culture-proven UTI in the elderly. Although it cannot be excluded that some of the patients presenting to the GP had a complicated UTI, the acute onset of symptoms, lack of systemic symptoms, or history of urological complaints suggests that the majority of men had cystitis. ${ }^{2}$ This diagnosis remains difficult, especially in men. In women, growth of $\geq 10^{5} \mathrm{CFU} / \mathrm{mL}$ is usually considered a positive urine culture. In well-instructed collection of clean midstream urine, lower growth could be considered positive in females. The anatomy of the male urethra makes the midstream urine probably unnecessary. ${ }^{11}$ The Dutch guidelines advise to use growth of $\geq 10^{3} \mathrm{CFU} / \mathrm{mL}$ as a positive urine culture in men. ${ }^{[16] \text { and }[17]}$

E. coli is the important uropathogen in the general population. ${ }^{[12]}$ and ${ }^{[18]}$ In females of all age categories, $E$. coli is the most frequently isolated uropathogen. ${ }^{[9],[10] \text { and }[19]}$ In accordance with the literature, we found that E. coli causes most male UTIs, followed by other enterobacteriaceae and enterococci ${ }^{[1]}{ }^{[2]}{ }^{[2]}$ whereas in female UTIs, Proteus mirabilis was more frequently isolated in the younger and K. pneumoniae in the elderly patients ${ }^{10}$; in elderly males, isolation of $P$. aeruginosa increased.

The diagnosis of a UTI in male patients is not always straightforward. Previously, we showed that a positive nitrite test has a high probability of disease (96\%) in male UTIs, and empirical antibiotic treatment should be considered awaiting culture results. ${ }^{6}$ The choice of empirical therapy depends on the distribution of uropathogens and their antibiotic susceptibility patterns. Most studies on uropathogens and their 
Koeijers, J.J., Verbon, A., Kessels, A.G.H., Bartelds, A., Donker, G., Nys, S., Stobberingh, E.E. Urinary tract infection in male general practice patients: uropathogens and antibiotic susceptibility. Urology: 2010

susceptibility have been performed in female patients with UTIs ${ }^{[7],[8],[9] \text { and }[10]}$ or a case mix of female and male patients. ${ }^{[12], ~[20], ~[21] ~ a n d ~[22] ~}$ In other studies describing uropathogens in men with symptoms indicative of UTI, $40 \%$ had negative urine cultures, E. coli was isolated in half of the UTIs, and Gram-positive cocci caused $20 \%$ of the UTIs, ${ }^{[5]}$ and ${ }^{[12]}$ which is comparable to our results. Twenty-four percent of men with culture-proven UTI were not treated at the first consultation on clinical grounds, suggesting that additional diagnostic tests are needed.

In The Netherlands, females with an uncomplicated UTI originating from the same population that we studied showed the lowest susceptibility percentages for the 3 most frequently isolated uropathogens $(E$. coli, P. mirabilis, and K. pneumoniae) for amoxicillin and trimethoprim, and more then $96 \%$ of the E. coli isolates were susceptible for amoxicillin-clavulanic acid, fluoroquinolones, and nitrofurantoin. ${ }^{[9]}{ }^{\text {and }}[10] \mathrm{In}$ male patients with UTI, deriving from the same GPs in the same period, the highest susceptibility rates were also found for amoxicillin-clavulanic acid, nitrofurantoin and fluoroquinolones (this study), suggesting that uropathogens in community-acquired UTIs have a similar susceptibility pattern in male and female patients. This supports the choice for the same empiric antibacterial therapy for symptoms of an uncomplicated UTI in both male and female patients.

Antibiotic resistance has become a consideration in the treatment of community-acquired UTIs. ${ }^{[23] \text { and }[24]}$ To our knowledge, sparse studies on antibiotic resistance in male UTIs have been reported. ${ }^{5}$ In a population study in Spain, including both complicated and uncomplicated UTIs in male and female patients, the susceptibility percentages for E. coli were low for amoxicillin (41\%), trimethoprim-sulfamethoxazole (66\%), and ciprofloxacin (77\%). ${ }^{12}$ In Spain and Latin American men with uncomplicated UTIs, the susceptibility percentages for $E$. coli were lower for trimethoprim-sulfamethoxazole and fluoroquinolones compared with our Dutch population, ${ }^{[12]}$ and [18] and fluoroquinolone resistance increased with age ${ }^{21}$ In Europe, E. coli, susceptibility for multiple drugs varied from $9 \%-40 \%$ in uncomplicated UTIs ${ }^{[7]}$ and ${ }^{[25]}$; in women, resistance to fluoroquinolones varied from $9 \%-16 \%,{ }^{[7]}$ and ${ }^{[25]}$ and in men it was as high as $25 \%{ }^{25}$ However, these susceptibility percentages may be higher than in the community because most cultures are done when treatment fails or in the case of complicated UTI. ${ }^{26}$ Because susceptibility varies with geographic region and population (nosocomial or community), empiric antibiotic prescription should be dependent on the susceptibility percentages of a specific community over time ${ }^{[12], \text {, [21], [27], [28], [29] and [30] }}$

In contrast to female patients, ${ }^{[9]}$ and $[10]$ the GP did not rely on the outcome of the nitrite dipstick test for the prescription of antimicrobial therapy, because only $47 \%$ of the treated patients had a positive dipstick test. Blinded for the culture results, the decision to treat was based on clinical grounds. The most prescribed antibiotics in all age groups were fluoroquinolones, trimethoprim-sulfamethoxazole, nitrofurantoin, and amoxicillin-clavulanic acid. Given the susceptibility percentages, it is justifiable that amoxicillin and trimethoprim are prescribed in the minority of patients. The prescription of nitrofurantoin is according to guidelines that state that males without a previous history of UTIs, systemic complaints, or signs of tissue invasion could be treated with nitrofurantoin. ${ }^{[16]}$ and ${ }^{[17]}$ Both the choice of antibiotic and duration of therapy indicate that patients were treated for uncomplicated UTIs, because treatment for complicated UTI favors the choice of fluoroquinolones and a longer duration of therapy. ${ }^{2}$

\section{CONCLUSIONS}

In the adult male presenting to the GP with acute symptomatic complaints of uncomplicated UTI, E. coli and other Gram-negatives are the most prevalent uropathogens. Empirical treatment in a male with suspected UTI may be started awaiting culture results based on susceptibility rates for uropathogens in the geographic region. Because susceptibility rates seem to be similar in community-acquired male and female UTIs, these rates may be obtained in either male or female UTIs. In elderly male patients with a suspicion of $P$. aeruginosa as the causative uropathogen, a fluoroquinolone should be given.

\section{REFERENCES}

1 B.A. Lipsky, Urinary tract infections in men: Epidemiology, pathophysiology, diagnosis, and treatment, Ann Intern Med 110 (1989), pp. 138-150.

2 B.A. Lipsky, Prostatitis and urinary tract infection in men: what's new; what's true?, Am J Med 106 (1999), pp. 327-334.

3 T.L. Griebling, Urologic diseases in America project: trends in resource use for urinary tract infections in men, J Urol 173 (2005), pp. 1288-1294. 
Koeijers, J.J., Verbon, A., Kessels, A.G.H., Bartelds, A., Donker, G., Nys, S., Stobberingh, E.E. Urinary tract infection in male general practice patients: uropathogens and antibiotic susceptibility. Urology: 2010

4 M.M. Collins, R. S. Stafford, M.P. O'Leary and M.J. Barry, How common is prostatitis?: A national survey of physician visits, J Urol 159 (1998), pp. 1224-1228. )

5 E. Hummers-Pradier, A.M. Ohse, M. Koch, W.R. Heizmann and M.M. Kochen, Urinary tract infection in men, Int J Clin Pharmacol Ther 42 (2004), pp. 360-366. V

6 J.J. Koeijers, A.G. Kessels and S. Nys et al., Evaluation of the nitrite and leukocyte esterase activity tests for the diagnosis of acute symptomatic urinary tract infection in men, Clin Infect Dis 45 (2007), pp. 894896.

7 E. Hummers-Pradier, M. Koch, A.M. Ohse, W.R. Heizmann and M.M. Kochen, Antibiotic resistance of urinary pathogens in female general practice patients, Scand J Infect Dis 37 (2005), pp. 256-261.

8 K. Gupta, D.F. Sahm, D. Mayfield and W.E. Stamm, Antimicrobial resistance among uropathogens that cause community-acquired urinary tract infections in women: a nationwide analysis, Clin Infect Dis 33 (2001), pp. 89-94.

9 S. Nys, T. van Merode, A.I. Bartelds and E.E. Stobberingh, Urinary tract infections in general practice patients: diagnostic tests versus bacteriological culture, J Antimicrob Chemother 57 (2006), pp. 955-958.

10 S. Nys, T. van Merode, A.I. Bartelds and E.E. Stobberingh, Antibiotic treatment and resistance of unselected uropathogens in the elderly, Int J Antimicrob Agents 27 (2006), pp. 236-241.

11 B.A. Lipsky, R.C. Ireton, S.D. Fihn, R. Hackett and R.E. Berger, Diagnosis of bacteriuria in men: specimen collection and culture interpretation, J Infect Dis 155 (1987), pp. 847-854.

12 A. Andreu, J.I. Alos, M. Gobernado, F. Marco, M. de la Rosa and J.A. Garcia-Rodriguez, [Etiology and antimicrobial susceptibility among uropathogens causing community-acquired lower urinary tract infections: a nationwide surveillance study], Enferm Infecc Microbiol Clin 23 (2005), pp. 4-9.

13 NCCLS, National Committee for clinical laboratory standards. Methods for Dilution Antimicrobial Susceptibility Tests for Bacteria That Grow Aerobically-Fifth Edition: Approved Standard, NCCLS, Villanova, PA, United States (2002) M7-A5.

14 P. Boyle, C. Robertson and C. Mazzetta et al., The prevalence of lower urinary tract symptoms in men and women in four centres: The UrEpik study, BJU Int 92 (2003), pp. 409-414.

15 G.S. Sonke, D. Kolman, J.J. de la Rosette, L.H. Donkers, P. Boyle and L.A. Kiemeney, [Prevalence of lower urinary tract symptoms in men and its influence on their quality of life: Boxmeer Study], Ned Tijdschr Geneeskd 144 (2000), pp. 2558-2563.

16 S.E. Geerlings, P.J. van den Broek and E.P. van Haarst et al., [Optimisation of the antibiotic policy in the Netherlands: X. The SWAB guideline for antimicrobial treatment of complicated urinary tract infections], Ned Tijdschr Geneeskd 150 (2006), pp. 2370-2376.

17 van Haaren K, VH, van Vliet S, Timmermans AE, Yadava R, Geerlings SE, Riet Ter G, van Pinxteren B. NHG-Standaard Urineweginfecties (tweede herziening). Huisarts Wetenschap. 2005;48:341-352.

18 A.C. Gales, H.S. Sader and R.N. Jones, Urinary tract infection trends in Latin American hospitals: report from the SENTRY antimicrobial surveillance program, Diagn Microbiol Infect Dis 44 (1997), pp. 289-299.

19 Nys S. Antibiotic resistance and commensal flora. In: Microbiology. University Maastricht, Maatricht The Netherlands; 2005:142.

20 K. Gupta, T.M. Hooton and W.E. Stamm, Increasing antimicrobial resistance and the management of uncomplicated community-acquired urinary tract infections, Ann Intern Med 135 (2001), pp. 41-50.

21 J.I. Alos, M.G. Serrano, J.L. Gomez-Garces and J. Perianes, Antibiotic resistance of Escherichia coli from community-acquired urinary tract infections in relation to demographic and clinical data, Clin Microbiol Infect 11 (2005), pp. 199-203.

22 L. Nesher, V. Novack, K. Riesenberg and F. Schlaeffer, Regional community-acquired urinary tract infections in Israel: diagnosis, pathogens, and antibiotic guidelines adherence: a prospective study, Int J Infect Dis 11 (2007), pp. 245-250.

23 L.E. Nicolle, Resistant pathogens in urinary tract infections, J Am Geriatr Soc 50 (2002), pp. S230S235.

24 L.E. Nicolle, Urinary tract infection: traditional pharmacologic therapies, Am J Med 113 (Suppl 1A) (2002), pp. 35S-44S.

25 A. Mahamat, J.P. Lavigne, P. Fabbro-Peray, J.M. Kinowski, J.P. Daures and A. Sotto, Evolution of fluoroquinolone resistance among Escherichia coli urinary tract isolates from a French University Hospital: application of the dynamic regression model, Clin Microbiol Infect 11 (2005), pp. 301-306.

26 T.J. Wiersma, [Guidelines for diagnosing urinary tract infections: advantages and disadvantages of various methods], Ned Tijdschr Geneeskd 145 (2001), pp. 720-726.

27 D. Prais, R. Straussberg, Y. Avitzur, M. Nussinovitch, L. Harel and J. Amir, Bacterial susceptibility to oral antibiotics in community acquired urinary tract infection, Arch Dis Child 88 (2003), pp. 215-218.

28 A.J. Matute, E. Hak and C.A. Schurink et al., Resistance of uropathogens in symptomatic urinary tract infections in Leon, Nicaragua, Int J Antimicrob Agents 23 (2004), pp. 506-509. 
29 J.A. Dromigny, B. Ndoye, E.A. Macondo, P. Nabeth, T. Siby and J.D. Perrier-Gros-Claude, Increasing prevalence of antimicrobial resistance among Enterobacteriaceae uropathogens in Dakar, Senegal: a multicenter study, Diagn Microbiol Infect Dis 47 (2003), pp. 595-600.

30 J.W. Warren, E. Abrutyn, J.R. Hebel, J.R. Johnson, A.J. Schaeffer and W.E. Stamm, Guidelines for antimicrobial treatment of uncomplicated acute bacterial cystitis and acute pyelonephritis in women: Infectious Diseases Society of America (IDSA), Clin Infect Dis 29 (1999), pp. 745-758.

\section{TABLES AND FIGURE}

Table 1. Percentage of isolated uropathogens found according to the age category

\begin{tabular}{lcccc}
\hline & \multicolumn{3}{c}{ Age Category in Years } & \\
\cline { 2 - 4 } & $18-50$ & $51-70$ & $>70$ & Total \\
& $\mathrm{n}=68$ & $\mathrm{n}=90$ & $\mathrm{n}=78$ & $\mathrm{~N}=236$ \\
E. coli & 59 & 42 & 45 & 48 \\
P. mirabilis & 4 & 6 & 8 & 6 \\
K. pneumoniae & 0 & 3 & 4 & 3 \\
Pseudomonas & 1 & 2 & 13 & 6 \\
$\quad$ spp. & 9 & 6 & 5 & 6 \\
Acinetobacter spp. & 9 & 21 & 14 & 15 \\
Other gram neg. & 16 & 8 & 5 & 9 \\
Enterococcus & 1 & 12 & 6 & 7 \\
Other gram post. & 12 & & \\
\hline
\end{tabular}

$\mathrm{N}=$ total number of cases. 
Table 2. Susceptibility percentages of the uropathogens for the different antibiotics

\begin{tabular}{|c|c|c|c|c|c|c|c|}
\hline \multirow[b]{2}{*}{ Uropathogen } & \multirow[b]{2}{*}{$\mathrm{N} *$} & \multicolumn{6}{|c|}{ Antibiotic Susceptibility \% } \\
\hline & & AMX & AMC & NF & TMP & TMP-SMX & FQ \\
\hline E. Coli & 113 & 75 & 100 & 97 & 81 & 81 & 97 \\
\hline P. mirabilis & 14 & 93 & 100 & 0 & 50 & 86 & 100 \\
\hline K. pneumoniae & 6 & 0 & 100 & 100 & 83 & 83 & 100 \\
\hline E. faecalis & 22 & 100 & 100 & 100 & 82 & 91 & 55 \\
\hline$P$. aeruginosa & 13 & 15 & 15 & 0 & 0 & 0 & 100 \\
\hline Acinetobacter spp. & 15 & 73 & 87 & 33 & 20 & 93 & 100 \\
\hline Other gram neg ${ }^{\dagger}$ & 36 & 22 & 72 & 89 & 81 & 86 & 94 \\
\hline Other gram post ${ }^{\ddagger}$ & 17 & 47 & 100 & 100 & 77 & 94 & 71 \\
\hline All uropathogens & 236 & $63 \%$ & $90 \%$ & $82 \%$ & $70 \%$ & $85 \%$ & $91 \%$ \\
\hline
\end{tabular}

AMX = amoxicillin; AMC = amoxicillin-clavulanic acid; NF = nitrofurantoin; TMP = trimethoprim; TMP-SMX = trimethoprim-sulfamethoxazole; $\mathrm{FQ}$ = fluoroquinolone.

$* \mathrm{~N}=$ total numbers of cases.

${ }^{\dagger}$ Consisting of Klebsiella oxytoca, Enterobacter cloacae, Enterobacter aerogenes, Citrobacter koseri, Citrobacter freundii, Morganella morganii, Stenotrophomonas maltophilia, Serratia marcescens.

* Consisting of coagulase-negative Staphylococcus, Staphylococcus aureus, group B Streptococcu, Staphylococcus saprophyticus. 
Table 3. Percentage of patients that received antibiotic treatment shown per age category

\begin{tabular}{lcccc}
\hline & \multicolumn{3}{c}{ Age Category } & \\
\cline { 2 - 4 } Antibiotic & $18-50 \mathrm{y}$ & $51-70 \mathrm{y}$ & $>70 \mathrm{y}$ & Total \\
Treatment & $\mathrm{n}=86$ & $\mathrm{n}=99$ & $\mathrm{n}=68$ & $\mathrm{~N}=253$ \\
Amoxicillin & 1 & 3 & 0 & 2 \\
Co-amoxicillin & 10 & 12 & 10 & 11 \\
TMP-SMX* & 26 & 24 & 21 & 24 \\
Trimethoprim & 3 & 1 & 1 & 2 \\
Nitrofurantoin & 14 & 15 & 19 & 16 \\
Quinolones & 31 & 32 & 35 & 33 \\
Other & 14 & 12 & 13 & 13 \\
Total \% & 100 & 100 & 100 & 100 \\
\hline N = Total number of cases. & & \\
$*$ Trimethoprim-sulfamethoxazole.
\end{tabular}

Figure 1. Percentage of all patients with antibiotic therapy in that age category treated for a specified amount of days.

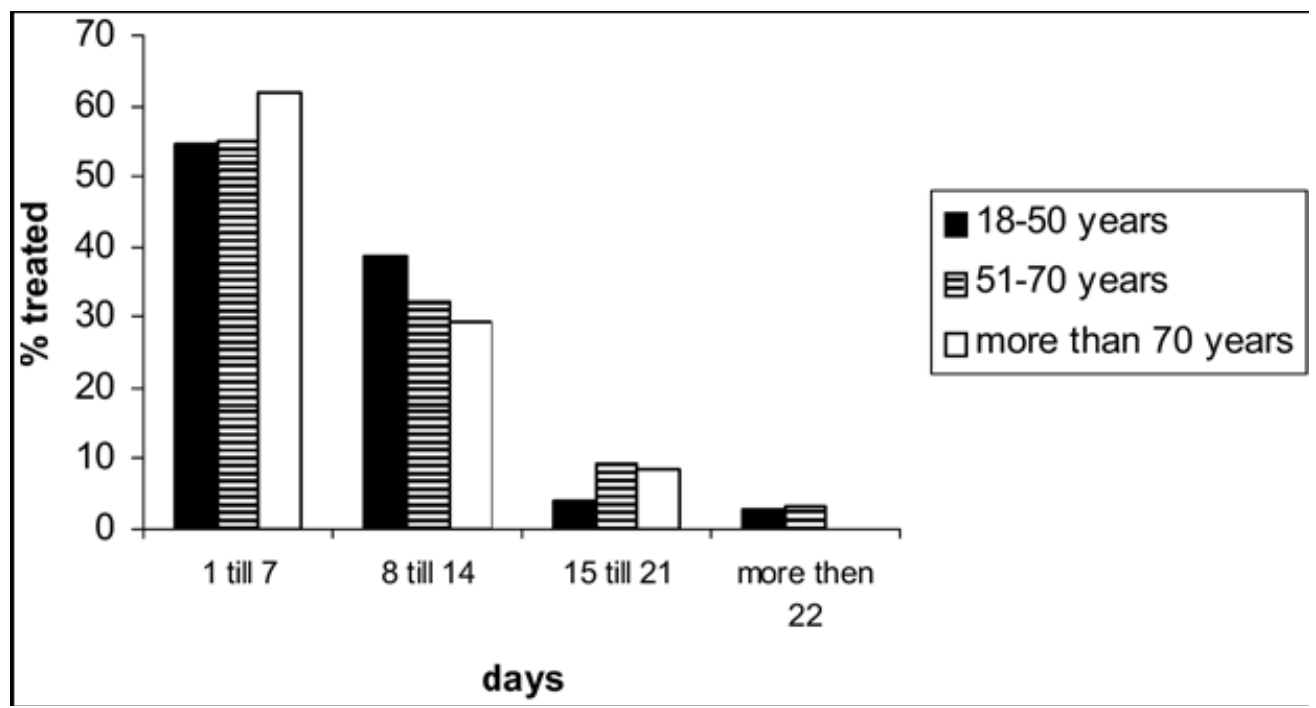

